J. Klin. Endokrinol. Stoffw. 2018 $111: 45-50$ https://doi.org/10.1007/s41969-018-0030-y Online publiziert: 8. Juni 2018

(c) Der/die Autor(en) 2018

CrossMark

Maximilian Zach ${ }^{1}$ Adelina Tmava-Berisha ${ }^{2}$ Stefan Schatzl ${ }^{3}$ - Michael Lehner ${ }^{4,5}$ • Greisa Vila ${ }^{6} \cdot$ Karin Amrein ${ }^{7,8}$

1 Medizinische Universität Graz, Graz, Österreich

${ }^{2}$ Klinische Abteilung für Endokrinologie u. Diabetologie, Medizinische Universität Graz, Graz, Österreich

${ }^{3}$ Univ. Klinik f. Innere Medizin I, Medizinische Universität Innsbruck, Innsbruck, Österreich

${ }^{4}$ Schilddrüsen- u. Osteoporosepraxis, Linz, Österreich

${ }^{5}$ Schilddrüsen- u. Osteoporosepraxis, Wels, Österreich

${ }^{6}$ Univ. Klinik f. Innere Medizin, Medizinische Universität Wien, Wien, Österreich

${ }^{7}$ Klinische Abteilung für Endokrinologie u. Diabetologie, Medizinische Universität Graz, Graz, Österreich

${ }^{8}$ Schilddrüsen/Endokrinologie/Osteoporose Institut Dobnig, Graz, Österreich

\title{
Die HypAus-Studie
}

\section{Erste Resultate einer multizentrischen österreichischen Beobachtungsstudie zu chronischem Hypoparathyreoidismus}

Hypo- als auch eine Hypermagnesiämie Ursache eines funktionellen Parathormonmangels mit Hypokalziämie sein [5].

Mit 70-80\% der Erkrankten kommt die Krankheit deutlich öfter bei Frauen vor [6], was darauf zurückzuführen ist, dass operative Eingriffe bei Schilddrüsenerkrankungen häufiger bei Frauen durchgeführt werden und auch da autoimmune Schilddrüsenerkrankungen wie z.B. der Morbus Basedow bei Frauen deutlich häufiger auftreten als bei Männern [7].

\section{Pathophysiologie und Symptome}

\section{Ätiologie}

Ätiologisch am häufigsten ist der postoperative Hypoparathyreoidismus (je nach Studie zwischen 66 und $78 \%$ ), welcher als Komplikation einer Thyreoidektomie (v.a. totale Thyreoidektomie), Parathyreoidektomie bzw. Neck Dissection auftreten kann. Die restlichen 25\% entfallen auf genetische, autoimmune oder idiopathische Ursachen, Infiltrationen der Nebenschilddrüsen im Rahmen von Systemerkrankungen oder Malignomen bzw. Ablagerungen von Eisen oder Kupfer in Folge von Speicherkrankheiten [4]. Zuletzt können sowohl eine
Durch den Wegfall der physiologischen PTH-Sekretion verliert der Körper vor allem in Situationen mit akut gesteigertem $\mathrm{Ca}^{2+}$-Bedarf die Möglichkeit, einen Abfall des $\mathrm{Ca}^{2+}$-Spiegels unter den Normbereich rasch durch eine $\mathrm{PTH}$-getriggerte $\mathrm{Ca}^{2+}$-Freisetzung aus dem Knochen zu kompensieren, was zur Entwicklung einer akuten Hypokalziämie führt. Durch den zusätzlichen Ausfall der Stimulation der 1-a-Hydroxylase (verminderte Bildung von Kalzitriol in der Niere und dadurch verminderte intestinale $\mathrm{Ca}^{2+}-\mathrm{Re}$ sorption) und den verstärkten renalen $\mathrm{Ca}^{2+}$-Verlust durch den Ausfall der PTH-
Wirkung an der Niere kommt es langfristig zur Ausbildung einer chronischen Hypokalziämie mit den entsprechenden Symptomen. Zuletzt kommt es durch den Ausfall der PTH-Wirkung zu einer gesteigerten renalen Phosphat-Reabsorption. Durch die Verbindung von Phosphat mit ionisiertem Kalzium kommt es zu einem Anstieg des Kalzium-PhosphatProdukts mit der Ausbildung von ektopen Kalzifizierungen. Typische Symptome und Langzeitkomplikationen werden in $\bullet$ Tab. 1 dargestellt.

Eine aktuelle Datenanalyse aus dem schwedischen Gesundheitsregister zeigte, dass es nach totalen Thyreoidektomien bei benigner Indikation in 5,2 \% der Fälle zum Auftritt eines permanenten Hypoparathyreoidismus kam. Zudem zeigte sich, dass diese Gruppe im Beobachtungszeitraum ein deutlich höheres Mortalitätsrisiko aufweist (Hazard Ratio [HR]: 2,09) [8].

Viele Patienten beklagen unspezifische Symptome wie Muskelschwäche, rezidivierende Muskelkrämpfe oder Parästhesien, was die Diagnosestellung mitunter verzögern kann. Bei einem klinischen Verdacht auf Hypoparathyreoidismus bzw. eine Hypokalziämie empfiehlt sich die Testung auf das Vorliegen eines Chvostek- oder Trousseau-Zeichens. Beim Chvostek-Zeichen kommt 
Tab. 1 Symptome u. Komplikationen des chronischen Hypoparathyreoidismus. (Modifiziert aus [13])

\begin{tabular}{|c|c|}
\hline Organsystem & Symptome bzw. Manifestationen \\
\hline \multirow[t]{8}{*}{ Neuromuskulär } & Müdigkeit \\
\hline & Muskelschwäche \\
\hline & Muskelkrämpfe (ev. schmerzhaft, Karpopedalspasmen) \\
\hline & Laryngospasmus, Stridor \\
\hline & Bronchospasmus, pfeifendes Atemgeräusch \\
\hline & Chvostek- u. Trousseau-Zeichen \\
\hline & EMG: rapid bursts (spontan oder durch Hyperventilation) \\
\hline & Erhöhte Kreatinkinase (CK) \\
\hline \multirow[t]{9}{*}{ Neurologisch, psychiatrisch } & Parästhesien (v. a. perioral u. an Akren) \\
\hline & Depressionen \\
\hline & Angststörungen \\
\hline & Persönlichkeitsstörungen \\
\hline & Krampfanfälle (Grand mal bzw. Petit mal) \\
\hline & Schlechtes Konzentrations- u. Erinnerungsvermögen („Brain fog“) \\
\hline & Basalganglienverkalkungen (Fahr-Syndrom) \\
\hline & Parkinson- u. Chorea-artige Symptome \\
\hline & Pseudotumor cerebri \\
\hline Lebensqualität & Vermindert (psychisch und physisch) \\
\hline \multirow[t]{4}{*}{ Kardiovaskulär } & Herzinsuffizienz \\
\hline & Angina pectoris \\
\hline & Arrhythmie und Reizleitungsstörungen \\
\hline & Verlängertes QTc-Intervall, EKG-Veränderungen \\
\hline \multirow[t]{3}{*}{ Renal } & Nephrokalzinose und Nierensteine (Hyperkalziurie) \\
\hline & Niereninsuffizienz \\
\hline & Erhöhtes Dialyserisiko \\
\hline \multirow[t]{4}{*}{ Ossär } & Erhöhte Knochendichte \\
\hline & Verminderter Knochenumbau \\
\hline & Schlechtere Mikroarchitektur (verminderte Knochenqualität) \\
\hline & Wirbelfrakturen \\
\hline \multirow[t]{2}{*}{ Ophthalmologisch } & Papillenödem \\
\hline & Katarakt, Korneaverkalkungen \\
\hline \multirow[t]{2}{*}{ Dermatologisch } & Alopezie, Schuppenbildung der Haut \\
\hline & Nageldeformitäten \\
\hline \multirow[t]{2}{*}{ Gastrointestinal } & Verstopfung, Steatorrhoe \\
\hline & Abdominale und biliäre Krämpfe \\
\hline \multirow[t]{4}{*}{ Dental } & Zementhyperplasie \\
\hline & Zahnschmelzhypoplasie \\
\hline & Agenesie von einem/mehreren Zähnen, kurze Zahnwurzeln \\
\hline & Erweiterung des Peridontalspalts \\
\hline Infektionen & Vermehrte Atemwegs- und Harnwegsinfektionen \\
\hline
\end{tabular}

es zu einer Kontraktion der ipsilateralen Gesichtsmuskulatur durch Beklopfen des N. facialis $2 \mathrm{~cm}$ vor dem Ohrläppchen. Beim Trousseau-Test wird durch Anlegen einer Blutdruckmanschette am Oberarm und dadurch ausgelöster Okklusion der empfiehlt sich für ein rasches Ergebnis zudem die Durchführung einer venösen Blutgasanalyse mit ionisiertem Kalzium.

Häufig wird die Einschränkung der Lebensqualität und Arbeitsfähigkeit unterschätzt. Hypoparathyreoidismus-Patienten haben unter Standardtherapie im Vergleich zur Normalbevölkerung eine deutlich verminderte Lebensqualität (durchschnittlich $24 \%$ geringer). Zudem ist die Arbeitsfähigkeit der Patienten eingeschränkt: Diese beziehen zehnmal öfter Sozialleistungen als die Normalbevölkerung ( $42 \%$ vs. $4 \%$ ) [3]. Häufig besteht ein sogenannter „empathy gap“ - die Bürde der Erkrankung wird unterschätzt. Oft werden die Beschwerden der Patienten auch fehlgedeutet oder nicht als Folge des PTH-Mangels erkannt $[10,11]$. Es empfiehlt sich, die jeweilige Symptomatik der Patienten ernst zu nehmen und diese als belastend anzuerkennen, dies kann die individuelle Krankheitsbewältigung verbessern [12].

Eine weitere Schwierigkeit ist diesbezüglich auch, dass der Hypoparathyreoidismus sich schleichend entwickeln kann und der postoperative auch mit einer deutlichen Latenzzeit zwischen Operation und Hypokalziämie bzw. Symptomen einhergehen kann. In einer retrospektiven Analyse von 105 postoperativen Hypoparathyreoidismus-Patienten am LKH Graz lag das Zeitintervall zwischen erster Schilddrüsenoperation und Diagnosestellung im Median bei 5,5 Jahren (eigene Daten, unpubliziert bzw. Diplomarbeit Martin Kern, Medizinische Universität Graz 2015).

\section{Diagnose}

Bei klinischem Verdacht auf Hypoparathyreoidismus oder unerklärter Hypokalziämie sollte eine laborchemische $\mathrm{Be}$ stimmung folgender Parameter erfolgen:

- intaktes PTH

- 25-(OH)-Vitamin D

- Albumin-korrigiertes Kalzium oder ionisiertes $\mathrm{Ca}^{2+}$

- Phosphat

- Magnesium

- Kreatinin

- (1,25-Dihydroxy-Vitamin D) 
Im 24-h-Harn (1x jährlich) sollte Folgendes bestimmt werden:

$$
\begin{aligned}
& \text { - Kalzium } \\
& \text { - Kreatinin mit Clearance } \\
& \text { - (Phosphat) }
\end{aligned}
$$

Die typische Laborkonstellation ergibt sich aus einer Hypokalziämie bei erniedrigten $(<15 \mathrm{pg} / \mathrm{ml})$ oder inadäquat normalen iPTH-Spiegeln und hoch normalen bis erhöhten Phosphatspiegeln. Zur Diagnosebestätigung sollte eine Hypokalziämie in Kombination mit einem inadäquat niedrigen $\mathrm{PTH}$-Spiegel $2 \mathrm{x}$ in einem Abstand von mindestens 2 Wochen gemessen werden.

Postoperativ empfiehlt sich die Bestimmung des iPTH am ersten postoperativen Tag. Eine retrospektive Analyse von Marcinkowska et al. ergab, dass ein iPTH-Spiegel $<5 \mathrm{pg} / \mathrm{ml}$ einen guten prognostischen Faktor für das Auftreten einer symptomatischen Hypokalziämie darstellt. Dadurch kann die entsprechende Therapie bereits vor einer klinisch manifesten Hypokalziämie begonnen werden [14].

\section{Therapie}

Die derzeitige Standardtherapie ist rein symptomatisch und erfolgt vor allem durch die orale Gabe von:

- Kalzium

- aktivem Vitamin D (Kalzitriol bzw. Analoga)

- nativem Vitamin D (Cholecalciferol/ Vitamin $\mathrm{D}_{3}$ bzw. Ergocalciferol/ Vitamin $\mathrm{D}_{2}$ )

Bei Bedarf können je nach Symptomatik ergänzend Magnesiumpräparate (bei Muskelkrämpfen) und Thiaziddiuretika (bei vermehrter Kalziurie) eingesetzt werden. Die jeweilige Dosierung der einzelnen Präparate orientiert sich primär an der individuellen Symptomausprägung und nicht an den Laborwerten. Dies soll einer Übersubstitution mit Kalzium und den damit verbundenen negativen Auswirkungen auf Nieren und andere Organe entgegenwirken. Eine rezente Analyse der dänischen Kohorte zeigte, dass eine Störung der KalziumPhosphat-Homöostase langfristig mit einem erhöhten Auftreten von renalen

J. Klin. Endokrinol. Stoffw. 2018 · 11:45-50 https://doi.org/10.1007/s41969-018-0030-y (c) Der/die Autor(en) 2018

\section{Zach · A. Tmava-Berisha · S. Schatzl · M. Lehner · G. Vila · K. Amrein \\ Die HypAus-Studie. Erste Resultate einer multizentrischen österreichischen Beobachtungsstudie zu chronischem Hypoparathyreoidismus}

\section{Zusammenfassung}

Der Hypoparathyreoidismus ist die letzte klassische, nicht mit dem fehlenden Hormon behandelte Endokrinopathie. Die Erkrankung tritt am häufigsten postoperativ auf und äußert sich oft durch die klinische Manifestation einer Hypokalziämie, jedoch kann sich dem behandelnden Arzt ein breites Spektrum an teils sehr unspezifischen Symptomen präsentieren. Der chronische Hypoparathyreoidismus führt in vielen Fällen zu einer deutlich eingeschränkten Lebensqualität.

Die derzeitige Standardtherapie ist rein symptomatisch und erfolgt vor allem durch die orale Gabe von Kalzium sowie aktivem und nativem Vitamin D. Eine Parathormonersatztherapie zeigte in verschiedenen Studien bereits gute Ergebnisse, wird derzeit aber aufgrund der hohen Therapiekosten kaum eingesetzt.

Im Rahmen der HypAus-Studie wurden Patienten mit chronischem Hypoparathyreoidismus hinsichtlich ihrer Ätiologie, Symptomatik, Therapie, und Lebensqualität evaluiert, zudem wurden einige Probanden einer neurokognitiven Testung unterzogen. Erste Ergebnisse deuten auf eine Einschränkung der Lebensqualität und eine Tendenz zur kognitiven Einschränkung.

\section{Schlüsselwörter}

Hypoparathyroidismus - Postoperative Komplikationen · Hypokalziämie . Hyperphosphatämie · Lebensqualität

\section{The HypAus study. First results of an Austrian multicentric observational study on chronic hypoparathyroidism}

Abstract

Hypoparathyroidism represents the last endocrine disorder that is not treated by the replacement of the missing hormone. The disease occurs mostly postoperatively and often manifests itself through hypocalcemia, though it can also present a wide spectrum of symptoms. Many patients suffer from an impaired quality of life. The current standard treatment is focused on controlling symptoms by oral administration of calcium and active and native vitamin D. Recent studies have suggested positive effects of parathormone replacement therapy, but due to its high cost it is currently not often used in clinical

und kardiovaskulären Komplikationen, Infektionen sowie einer erhöhten Mortalität assoziiert ist [15].

\section{HypAus-Studie}

Ziel dieser prospektiven multizentrischen Beobachtungsstudie ist der Aufbau einer Kohorte von an chronischem Hypoparathyreoidismus erkrankten $\mathrm{Pa}$ tienten. Die Identifikation der Patienten erfolgt an der Medizinischen Universität Graz, der Medizinischen Universität practice. In the course of the HypAus-Study, patients with chronic hypoparathyroidism have been evaluated regarding their etiology, symptoms, therapy and quality of life. In addition, some subjects have undergone neurocognitive testing. Preliminary results indicate a reduced quality of life and a tendency towards cognitive impairment.

\section{Keywords}

Hypoparathyroidism · Complications, postoperative - Hypocalcemia $\cdot$ Hyperphosphatemia $\cdot$ Quality of life 
Tab. 2 Übersicht über die wichtigsten Charakteristika der bisher in die HypAUS Studie eingeschlossenen Patienten

Altersschnitt (Jahre)
Patienten (w/m)
Alter bei Diagnosestellung (Jahre, median)
Mittlere Erkrankungsdauer (Jahre)
Ätiologie

Lebensqualität (QoL)

Symptome u. Komplikationen

Parästhesien

50

30 (21/9 entspr. $70 / 30 \%)$

$45,5$ (min: $5, \max : 68)$

9

Postoperativ: $83 \%$

Genetisch: $10 \%$

Idiopathisch: $7 \%$

Körperliche Summenskala: $-8,9 \%$ d. Norm

Psychische Summenskala: $-17,7 \%$ d. Norm

Muskelkrämpfe

$26(87 \%)$

$22(73 \%)$

Myalgien

$8(27 \%)$

Tetanie (min 1x seit KH-Beginn)

$11(37 \%)$

Herzrhythmusstörungen (subjektiv)

$8(27 \%)$

Herzrhythmusstörungen (im EKG)

$1(3 \%)$

Hypotonie/Schwindel

$8(27 \%)$

Zahnprobleme

$11(37 \%)$

Erhöhtes Harnkalzium

$7(23 \%)$

Nephrokalzinose u. Nephrolithiasis

$1(3 \%)$

Stationärer Aufenthalt (alle Ind.)

$18(60 \%)$

Stationärer Aufenthalt (HPT-assoz.)

$7(23 \%)$

$17(57 \%)$

$3(10 \%)$

$5(17 \%)$

$8(27 \%)$

$5(17 \%)$

$2(7 \%)$

$11(37 \%)$

Infektionen

$3(10 \%)$

$7(23 \%)$

$11(37 \%)$

$3(10 \%)$

1 (3\%)

$5(17 \%)$

$3(10 \%)$

$3(10 \%)$

$9(30 \%)$

Verschlechterte Sehleistung

$w / m$ weiblich/männlich, KH-Beginn Krankheitsbeginn, Ind Indikation, HPT-assoz. Hypoparathyreoidismus-assoziiert, i.v. intravenös, CT Computertomografie

\section{Erste Ergebnisse}

Im Zeitraum Jänner bis Oktober 2017 konnten 30 Patienten für die HypAusStudie rekrutiert werden (Diplomarbeit Maximilian Zach, Medizinische Universität Graz 2017) (• Tab. 2).
$70 \%$ der Patienten sind weiblich, das mediane Alter bei Diagnosestellung liegt bei 46 Jahren bei einer mittleren Erkrankungsdauer von 9 Jahren. Ätiologisch an erster Stelle liegt in unserem Kollektiv mit $83 \%$ der postoperative Hypoparathyreoidismus, wobei bei all diesen Pati- enten eine totale Thyreoidektomie durchgeführt wurde. Häufigste Operationsindikation war eine Knotenstruma. Bei den verbleibenden 5 Patienten war die Ursache für die Erkrankung eine genetische Mutation bzw. ein Autoimmunprozess.

In puncto Lebensqualität lag das Gesamtkollektiv in der körperlichen Summenskala $8,9 \%$ unter der Norm, in der psychischen Summenskala 17,7\% unter der Norm bzw. dem Vergleichswert des steirischen Normkollektivs. Die Analyse der Lebensqualität bezogen auf die Erkrankungsdauer ergab keine direkte Korrelation der Lebensqualität mit der Dauer der Erkrankung. Ein interessanter Aspekt ergab sich bei der intraindividuellen Ausprägung der Einschränkung der Lebensqualität. Ein Großteil der Patienten empfand die subjektiv wahrgenommene Belastung durch die chronische Erkrankung entweder in der körperlichen oder in der psychischen Dimension deutlich stärker ausgeprägt.

Bei den Symptomen wurden klassische Symptome wie Parästhesien (87\%) und rezidivierende Muskelkrämpfe (73\%) am häufigsten angegeben. $37 \%$ erlitten mindestens einmal im Verlauf eine Tetanie und $57 \%$ benötigten mindestens einmal eine i.v.-Kalziuminfusion aufgrund ihrer ausgeprägten Hypokalziämie bzw. Symptomatik. Zudem zeigte sich eine deutliche Häufung von unspezifischen Symptomen und Langzeitkomplikationen wie Myalgien (27\%), Schwindelgefühl (27\%), Zahnproblemen $(37 \%)$, Atemnot (27\%), Depression $(37 \%)$, verschlechterte Sehleistung (30\%), Dysphagie (17\%), Ataxie und Dysarthrie (je 10\%) sowie dem Auftreten eines sogenannten „Brain fog“ (verminderte Konzentrations- und Erinnerungsleistung, $23 \%$ ).

Bei der Analyse der Laborwerte zeigten sich lediglich $46 \%$ der Patienten im gewünschten Zielbereich bezüglich des Gesamtkalziums, die restlichen Ergebnisse zeigten eine Hypo- oder Hyperkalziämie. Beim ionisierten Kalzium waren zum Messzeitpunkt $94 \%$ der Patienten hypokalziämisch. 


\section{HypAus Neurokognitive Testung}

Ein besonderer Teil der HypAus-Studie ist die Exploration der neurokognitiven Ebene bei Patienten mit chronischem Hypoparathyreoidismus. Unser Ziel ist, die Prävalenz der neurokognitiven Dysfunktion bei chronischem Hypoparathyreoidismus zu bestimmen, mögliche Unterschiede in der kognitiven Funktion zu erkennen und die Korrelation von $\mathrm{Hy}$ poparathyreoidismus und Major Depression zu untersuchen.

Eine neurokognitive Testbatterie mit Fokus auf exekutive Funktionen, verbales und figurales Gedächtnis, Einschätzung des Intelligenzquotienten (IQ), Lebensqualität und Schlafqualität wird eingesetzt. Als illustratives Beispiel werden die Ergebnisse von 3 Tests bei den ersten 10 Patienten dargestellt.

Das Beck-Depressions-Inventar (BDI-II) ist ein Testverfahren, das den Schweregrad einer depressiven Symptomatik erfasst. Unsere Stichprobe erreichte im Durchschnitt einen Depressionsscore von 11 Punkten, was einer leichten depressiven Symptomatik entspricht. Zudem wurde die Mini Mental State Examination (MMSE) verwendet, um kognitive Defizite zu erkennen. Hier ergab sich ein Medianwert von 28,5, dieser liegt nur knapp unter dem Medianwert der Normalbevölkerung $(M=29)$. Die Fähigkeit zur Symbolerkennung sowie die allgemeine Informationsverarbeitungsgeschwindigkeit wurden mittels Trail-Making-Test A und B untersucht. Zur Aufgabenlösung des ersten Teils benötigten die Patienten durchschnittlich 37,5s (der Durchschnittswert der Normalbevölkerung liegt bei $30 \mathrm{~s}$ ). Für den zweiten Teil brauchten die Patienten im Durchschnitt 92,50s (Mittelwert Normalbevölkerung $=75 \mathrm{~s}$ ), somit scheint sowohl beim Teil A als auch beim Teil B eine verlangsamte Aufgabenlösung unserer Patienten vorzuliegen.

Es scheint somit bei chronischem Hypoparathyreoidismus eine Tendenz zur kognitiven Einschränkung vorzuliegen, jedoch sind sowohl die Stichprobe als auch die Anzahl der beschriebenen Tests derzeit noch sehr klein. Eine detaillierte Beschreibung unserer Studie mit einer 
größeren Anzahl der Patienten wird zu einem späteren Zeitpunkt durchgeführt.

\section{Ausblick}

Die Auswertung der ersten Daten zeigte, dass Patienten mit Hypoparathyreoidismus in vielen Fällen trotz (symptomatischer) Therapie eine deutliche Symptomatik und Belastung präsentieren, die von Außenstehenden gerne unterschätzt wird. Der Einsatz einer kausalen Therapie könnte hier einen großen Schritt in Richtung einer verbesserten Lebensqualität zeigen.

Eine sonst in der Endokrinologie übliche Ersatztherapie des fehlenden Hormons ist in Österreich seit kurzem zwar zugelassen (Parathormon 1-84), wird aber aufgrund der hohen Behandlungskosten derzeit noch kaum eingesetzt. Der Einsatz von PTH (1-34) ist beim chronischen Hypoparathyreoidismus zwar möglich, stellt jedoch eine Off-LabelSituation dar. Die Wirkung des PTHErsatzes wurde in einigen Studien bereits nachgewiesen, und er ist in den USA bereits seit 2015 zugelassen. Mittlerweile werden dort mehrere hundert Patienten damit behandelt $[16,17]$.

Offen bleibt, ob eine PTH-Kontrolle nach Schilddrüsenoperationen über einen längeren Zeitraum (derzeit $6 \mathrm{Mo}$ nate) sinnvoll wäre, um Patienten mit verzögertem Krankheitsbeginn frühzeitig zu erkennen und dementsprechend rascher einer adäquaten Therapie zuzuführen.

Es scheint bei chronischem Hypoparathyreoidismus eine Tendenz zur kognitiven Einschränkung vorzuliegen.

\section{Korrespondenzadresse}

\section{Assoz. Prof. PD Dr. K. Amrein, MSc \\ Klinische Abteilung für Endokrinologie $u$. Diabetologie, Medizinische Universität Graz Auenbruggerplatz 15, $8036 \mathrm{Graz}$, Österreich karin.amrein@medunigraz.at}

Funding. Open access funding provided by Medical University of Graz.

\section{Einhaltung ethischer Richtlinien}

Interessenkonflikt. K. Amrein erhielt Honorare von Shire für Vorträge und Tätigkeit im Advisory Board. M. Zach, A. Tmava-Berisha, S. Schatzl, M. Lehner und G. Vila geben an, dass kein Interessenkonflikt besteht.

Alle beschriebenen Untersuchungen am Menschen wurden mit Zustimmung der zuständigen Ethik-Kommission, im Einklang mit nationalem Recht sowie gemäß der Deklaration von Helsinki von 1975 (in der aktuellen, überarbeiteten Fassung) durchgeführt. Von allen beteiligten Patienten liegt eine Einverständniserklärung vor.

Open Access. Dieser Artikel wird unter der Creative Commons Namensnennung 4.0 International Lizenz (http://creativecommons.org/licenses/by/4.0/deed de) veröffentlicht, welche die Nutzung, Vervielfältigung, Bearbeitung, Verbreitung und Wiedergabe in jeglichem Medium und Format erlaubt, sofern Sie den/die ursprünglichen Autor(en) und die Quelle ordnungsgemäßnennen, einen Linkzur Creative Commons Lizenz beifügen und angeben, ob Änderungen vorgenommen wurden.

\section{Literatur}

1. Underbjerg L, Sikjaer T, Mosekilde L, Rejnmark L (2013) Cardiovascular and renal complications to postsurgical hypoparathyroidism: a Danish nationwide controlled historic follow-up study. JBone Miner Res 28(11):2277-2285

2. Powers J, Joy K, Ruscio A, Lagast H (2013) Prevalence and incidence of hypoparathyroidism in the United States using a large claims database. JBone Miner Res 28(12):2570-2576

3. Astor MC, Lovas K, Debowska A, Eriksen EF, Evang JA, Fossum Cet al (2016) Epidemiology and healthrelated quality of life in hypoparathyroidism in Norway.JClinEndocrinol Metab 101(8):3045-3053

4. Clarke BL, Brown EM, Collins MT, Juppner $H$, Lakatos P, Levine MA et al (2016) Epidemiology and diagnosis of hypoparathyroidism.J Clin Endocrinol Metab 101(6):2284-2299

5. Brandi ML, Bilezikian JP, Shoback D, Bouillon R, Clarke BL, Thakker RV et al (2016) Management of hypoparathyroidism: summary statement and guidelines. J Clin Endocrinol Metab 101(6):2273-2283

6. Bilezikian JP, Khan A, Potts JT Jr., Brandi ML, Clarke BL, Shoback D et al (2011) Hypoparathyroidism in the adult: epidemiology, diagnosis, pathophysiology, target-organ involvement, treatment, and challenges for future research. J Bone Miner Res 26(10):2317-2337

7. Herold G (2011) Herold Innere Medizin

8. Almquist M, Ivarsson K, Nordenstrom E, Bergenfelz A (2018) Mortality in patients with permanent hypoparathyroidism after total thyroidectomy. $\mathrm{Br} J$ Surg.https://doi.org/10.1002/bjs.10843

9. Jesus JE, Landry A (2012) Chvostek's and Trousseau's Signs. N Engl J Med 367(11):e15

10. Cho NL, Moalem J, Chen L, Lubitz CC, Moore FD Jr., Ruan DT (2014) Surgeons and patients disagree on the potential consequences from hypoparathyroidism. Endocr Pract 20(5):427-446

11. Horvath K (2015) Hypoparathyroidism - The treatment paradox. Endocrine News 2015, S15-17

12. RejnmarkL (2018) Quality of life in hypoparathyroidism. Endocrine 59(2):237-238
13. Shoback DM, Bilezikian JP, Costa AG, Dempster D, Dralle H, Khan AA et al (2016) Presentation of hypoparathyroidism: etiologies and clinical features. JClin Endocrinol Metab 101(6):2300-2312

14. Marcinkowska $M$, Sniecikowska B, Zygmunt $A$, Brzezinski J, Dedecjus M, Lewinski A (2017) Postoperative hypoparathyroidism in patients after total thyroidectomy - retrospective analysis. Neuro Endocrinol Lett 38(7):488-494

15. Underbjerg L, Sikjaer T, Rejnmark L (2017) Longterm complications in patients with hypoparathy roidism evaluated by biochemical findings: a casecontrol study. J Bone Miner Res. https://doi.org/10. 1002/jbmr.3368

16. Rejnmark L, Underbjerg L, Sikjaer T (2015) Hypoparathyroidism: Replacement therapy with parathyroid hormone. Endocrinol Metab (Seoul) 30(4):436-442

17. Mannstadt $M$, Clarke BL, Vokes $T$, Brandi ML, Ranganath L, Fraser WD et al (2013) Efficacy and safety of recombinant human parathyroid hormone (1-84) in hypoparathyroidism (REPLACE): a double-blind, placebo-controlled, randomised, phase 3 study. Lancet Diabetes Endocrinol 1(4):275-283 